\title{
Mild head injuries: prospective study
}

\section{Nikolaos Syrmos*, Vasilios Valadakis, Kostantinos Grigoriou and Dimitrios Arvanitakis}

\author{
Address: Neurosurgical Department-Venizeleio General Hospita, Heraklion, Crete \\ * Corresponding author
}

from International Society on Brain and Behaviour: 3rd International Congress on Brain and Behaviour

Thessaloniki, Greece. 28 November - 2 December 2007

Published: 17 April 2008

Annals of General Psychiatry 2008, 7(SuppI I):S303 doi:I0.1 I86/I744-859X-7-SI-S303

This abstract is available from: http://www.annals-general-psychiatry.com/content/7/SI/S303

(C) 2008 Syrmos et al.; licensee BioMed Central Ltd.

\section{Background}

To define the incidence of positive CT scans in patients with different grades of mild head injury (MHI), 2. To identify clinical predictors of positive CT scans, 3 . To evaluate the usefulness of plain radiographs in the triage of patients with MHI, 4. To evaluate frequency and nature of surgical intervention in $\mathrm{MHI}, 5$. To evaluate the risk of deterioration in MHI, and 6. To find out whether patients with normal neurological examination and normal CT can be safely discharged.

\section{Materials and methods}

In a prospective study conducted over a period 1 year in our Department, all patients with mild head injury (defined as Glasgow Coma Scores (GCS) 13-15) were admitted to the head injury unit. Patients underwent standard clinical examination, skull radiography and cranial CT. No clinical criteria were used to select patients for CT scanning and all the patients were subjected to CT. Patients with negative findings on CT and a normal neurological examination were discharged after 24 hours of observation. Patients with positive findings on cranial CT were treated either medically or surgically as deemed necessary. Outcome measures included safe discharge, clinical deterioration, need for surgical intervention or death. The following factors were analyzed statistically to find out whether they can be used as predictive factors for positive cranial CT. They were: age, sex, mode of injury, loss of consciousness, post-traumatic seizures, ear/ nose/ throat bleeding, vomiting, admission GCS score, scalp injury, polytrauma, focal neurological deficit, fractures visualized on skull radiography.

\section{Results}

300 patients were included in the study. Of these males constituted $60 \%$, 40\% females. Age, mode of injury, loss of consciousness, post-traumatic seizures, ENT bleeding, vomiting, scalp injury and polytrauma were not found to be predictors of positive CT. Admission GCS score, focal neurological deficits, and fractures detected by skull radiography were found to be statistically significant predictors of positive findings on CT.

\section{Conclusions}

Patients with multiple lesions on CT had a higher chance of deterioration than those with single lesions. The duration of hospital stay was prolonged in patients with positive CT. As no patient with a normal neurological examination and a normal CT deteriorated, we believe these patients can be safely discharged without need for admission and observation.

\section{References}

I. Livingston $D H$, Loder PA, Hunt $C D$ : Minimal head injury: is admission necessary? Am Surg. I99।, 57(I): 14-7. Jan

2. Murshid WR: Management of minor head injuries: admission criteria, radiological evaluation and treatment of complications. Acta Neurochir (Wien) 1998, I40(1):56-64.

3. Borczuk P: Predictors of intracranial injury in patients with mild head trauma. Ann Emerg Med 1995, 25(6):731-6. Jun 